\title{
Aplicación de dos técnicas de recogida de datos para la realización de estudios de usuarios
}

\author{
Carmen Martín Moreno \\ Departamento de Biblioteconomía y Documentación, \\ Universidad Carlos III de Madrid C/ \\ Madrid 126, 28903 Getafe, Madrid (España) \\ E-mail: cmartin@ bib.uc3m.es \\ ElÍAS SANZ CASAdo \\ Departamento de Biblioteconomía y Documentación, \\ Universidad Carlos III de Madrid C/ \\ Madrid 126, 28903 G etafe, Madrid (España) \\ E-mail: elias@ bib.uc3m.es
}

\section{RESUMEN}

En el artículo se propone la realización de estudios de usuarios mediante la utilización de más de una técnica de recogida de datos con el fin de aumentar la validez y fiabilidad de los resultados. A través de un método directo y otro indirecto se obtuvieron una serie de indicadores sobre los hábitos y necesidades de información de un grupo de investigadores españoles en ciencias de la vida. D ado que los valores obtenidos en ambos casos fueron muy similares, se concluye que la metodología aplicada fue adecuada para el estudio y los resultados obtenidos son totalmente fiables.

Palabras clave: Estudios de Usuarios, Recopilación de datos.

\author{
APPlication OF TWO TECHNiQUES \\ FOR DATA COLLECTION IN USER STUDIES \\ CARMEn Martín-Moreno y Elías SANZ-CaSAdo
}

\begin{abstract}
AbST RACT
In this article user studies are proposed which make use of more than one technique for the data collection in order to increase the validity and reliability of results. By using both a direct and an indirect method a series of indicators were obtained on the information habits and needs of a group of Spanish researchers in the life sciences. Taking into consideration that values proved similar in both cases we can conclude that the applied method was suitable for the study and that results obtained are completely reliable.
\end{abstract}

Key words: User Studies, Data Colleting

$$
\begin{gathered}
\text { Trabajo recibido } \\
\text { el } 12 \text { de julio de } 2001 \\
* \\
\text { Trabajo aceptado } \\
\text { el } 15 \text { de julio de } 2001
\end{gathered}
$$




\section{INTRODUCCIÓN}

D ara poder resolver los problemas de información de los usuarios, los centros de información pueden llevar a cabo distintas acciones, entre las cuales destacan la realización de estudios de usuarios, pues permiten que los profesionales de los centros puedan inferir el uso y las necesidades reales de información que tienen sus usuarios. Este tipo de estudios constituyen una herramienta multidisciplinar vital para que los profesionales mejoren su conocimiento sobre el uso de la información y de su difusión (Siatri, 1999), pues permiten analizar diferentes aspectos y características de la relación que se establece entre la información y el usuario, empleando para ello distintas técnicas de investigación. La aplicación de distintas metodologías de recogida y tratamiento de datos, permite acceder a un modelo de comportamiento de los usuarios de un centro de información (entendiendo como usuarios, no sólo los que acuden a él, sino también los que potencialmente podrían hacerlo).

Durante un largo periodo de tiempo, los autores que hicieron revisiones bibliográficas sobre estudios de usuarios manifestaron quejas sobre el aspecto metodológico (Menzel, 1966; Herner y Herner, 1967; Paisley, 1968; Allen, 1969; 0 Crawford, 1978), coincidiendo en que la carencia de una metodología apropiada hacía muy difícil trabajar con una enorme diversidad de conceptos (necesidades, uso, comportamiento, hábitos, etc.) y tipos de estudios (comportamiento de los científicos ante la comunicación, flujo de la comunicación entre investigadores, uso de distintos servicios o de distintos materiales, etc.), pues para algunos autores "los estudios de usuarios, estudios de uso, estudios de necesidades de información, estudios de transferencia de información, estudios de comportamiento de la comunicación, estudios de la difusión y utilización de la información, investigación sobre el usuario, etc., son términos estrechamente relacionados y con frecuencia poco definidos" (Sridhar, 1995).

El estudio de las características de los hábitos y necesidades de información de los científicos experimentales españoles que trabajaban en el área de las Ciencias de la Vida, constituyó el trabajo de investigación de la tesis doctoral que presentó Martín Moreno (1999) en la Universidad Carlos III Madrid para obtener el grado de D octor. En dicho trabajo se utilizaron dos métodos de recogida de datos que permitieron determinar claramente las necesidades de información de estos científicos.

En general, la investigación tiende a centrarse más en los resultados e implicaciones que en los métodos utilizados, pese a que a aquéllos no se les puede otorgar credibilidad sin conocer la fiabilidad con que se han obtenido, esto es, sin saber si se han empleado las metodologías adecuadas (Hernon y Schwartz, 1994). Por ello, en este trabajo, aunque tendremos que hablar de algunas características del grupo de investigadores españoles estudiado, lo que realmente pretendemos es resaltar el interés de aplicar más de una técnica de recogida de datos en este tipo de estudios, 
ya que así se obtienen resultados más consistentes y con posibilidad de ser validados, evitándose además algunos sesgos.

En un campo verdaderamente multidisciplinar como la bibliotecología, que ha tenido que tomar prestados métodos desarrollados por otras disciplinas para impulsar su investigación, la aplicación de más de un método de estudio otorga mayor credibilidad a los resultados obtenidos.

\section{METOdOLOGÍA}

Como acabamos de indicar, en el desarrollo de las ciencias sociales, y de la bibliotecología como parte de ellas, coexisten alternativas metodológicas diversas de las cuales podemos hacer una primera diferenciación entre métodos cuantitativos y cualitativos.

Los métodos cuantitativos que se utilizan en el análisis sociológico, se corresponden con un único modelo general a todas las ciencias, estos es, descriptivo y basado en el contraste empírico y en la medición objetiva de los fenómenos sociales. En cambio, los cualitativos son más interpretativos, se basan en la observación y en la comunicación oral, y estudian aspectos muy puntuales de la vida social e incluso del individuo (Bryman, 1988; Glazier, 1992). Ambas perspectivas metodológicas difieren tanto en la estrategia seguida para recoger la información como en su análisis (Cooper, 1992; D ervin, 1992; G lazier, 1992).

A la hora de realizar un estudio de usuarios, el método de recogida de datos que se aplicará dependerá fundamentalmente de los objetivos trazados. En nuestro caso, deseábamos conocer un conjunto de características propias de un grupo concreto de usuarios que permitiera determinarlos de forma específica. Cuando se aborda el estudio de una realidad social, es más adecuado valerse de una pluralidad de vías compatibles y complementarias para evitar los sesgos propios de cada una y para asegurar una mejor consecución del objetivo propuesto (Paisley, 1968). Por todo ello, en nuestro análisis empleamos una combinación de técnicas cuantitativas que permitieran un mayor conocimiento de la población a estudiar. Al trabajar así cada una de las técnicas aporta datos específicos que pueden complementarse, permitiendo incluso que una de ellas confirme los datos obtenidos mediante la otra.

En dicho trabajo se utilizaron técnicas directas e indirectas, para disponer así de un mayor número de puntos de referencia y lograr mayor precisión en los objetivos propuestos. Con ello aumentamos la validez de los resultados y el grado de confianza en los mismos, entendiendo por validez la adecuación de un valor particular respecto a la realidad a la que apunta, y por grado de confianza la adecuación de las metodologías empleadas para su obtención.

Los métodos directos permiten analizar las características de los usuarios objeto del estudio a través de la información que aportan al responder a distintas 
preguntas planteadas (Sanz, 1994). Se utilizan cuando se quiere obtener una información profunda, que provenga directamente del sujeto analizado. La encuesta autoadministrada y enviada por correo es probablemente la técnica directa más socorrida para conocer las características y necesidades de información de muchos colectivos de usuarios, tal y como se desprende del trabajo de Julien (1996).

Los métodos indirectos permiten deducir las características de los usuarios a través de documentos generados con distintos fines, como pueden ser sus trabajos, o la información que demandan a una biblioteca o a un centro de documentación. El análisis de referencias y de los documentos solicitados son los que se utilizan con mayor frecuencia por su facilidad de aplicación y por la información que aportan (Sanz, 1994).

El objetivo de la tesis doctoral que dio origen a este trabajo (Martín Moreno, 1999), era conocer las características de los investigadores españoles de Ciencias de la Viday sus necesidades deinformación. Para conseguirlo, se aplicaron dos metodologías distintas en la recogida de información. Un análisis de las peticiones de documentos realizadas - al Servicio de Acceso al D ocumento Primario, del Centro de Información y D ocumentación Científica (CIND OC)- por investigadores españoles de Ciencias de la Vida y una encuesta autoadministrada y enviada por correo.

En el caso del análisis de demanda de documentos había que delimitar, en primer lugar, los centros que debían formar parte del estudio, para lo cual utilizamos como referente los recogidos en una base de datos suministrada por la Comisión Interministerial de Ciencia y Tecnología (CICYT), constituida por los investigadores que habían solicitado proyectos de investigación financiados por dicho organismo gubernamental español, y cuyo tema estuviera incluido en el código UNESCO "24", que integraba el área de nuestro interés. La base constaba de 4.342 registros, cada uno de los cuales recogía, entre otros, los nombres y apellidos de los investigadores, los códigos UNESCO de sus trabajos, el departamento o centro de trabajo, la institución a que pertenecían y su dirección postal.

Una vez determinados los centros de investigación, se procedió a la creación de una base de datos que nos permitiera el análisis posterior de las peticiones de información realizadas al CIND OC durante el período comprendido entre los años 1988 y 1993.

Una vez introducidos en la base de datos los 43.468 documentos válidos de que disponíamos, hubo que realizar una labor de depuración, codificación y normalización de algunos campos, con objeto de poder clasificar la información y obtener indicadores bibliométricos.

El método directo aplicado para conocer las características de los científicos españoles como usuarios de la información, fue una encuesta autoadministrada y enviada por correo postal. Este método de recogida de datos se caracteriza por ser el propio encuestado el que lee el cuestionario y anota las respuestas. El instrumento que se aplicó fue un cuestionario estructurado, preparado exclusivamente para este fin, por lo que las preguntas se realizaron conforme a objetivos. 
La muestra a la que dirigimos la encuesta estaba integrada por el 50\% de los investigadores que aparecían en la base de datos proporcionada por la CICYT. Se utilizó un muestreo probabilístico de tipo aleatorio simple. La tasa de respuesta obtenida fue del 18.24\% (396), similar a la de otros autores (Cronin, 1980; Chau, 1994; Tillotson, e. al, 1995; o Blanchard y G iustini, 1997), lo que permitió estudiar las características y necesidades de información de estos científicos.

Una vez recogida la información de los cuestionarios recibidos, los campos creados se normalizaron y se trabajaron en Excel 7.0, lo que permitió el estudio y valoración de las distintas variables independientes con las que se ha trabajado. Tras ser tabuladas las respuestas, se emplearon programas informáticos para realizar diferentes pruebas estadísticas y así establecer relaciones entre ellas.

A partir de los datos recogidos, independientemente del método empleado para ello, se obtuvieron distintos indicadores bibliométricos, "datos numéricos extraídos de los documentos que publican los investigadores o de los que son solicitados a la biblioteca, y que permiten analizar distintas características de las necesidades y demandas de información existentes dentro de la comunidad de usuarios que la visitan" (Sanz y Martín, 1998). Sin embargo, hay que tener en cuenta que cuando se utilizan dos métodos, la principal diferencia radica en la forma de obtener los indicadores. En el análisis de demandas, su valor deviene de la información que aparece en los documentos de petición de información, y en la encuesta se calculan a partir de las respuestas que los usuarios señalan en el cuestionario.

Los indicadores bibliométricos pueden ser unidimensionales y multidimensionales. Los primeros estudian una sola característica de los documentos, o de los usuarios que se están estudiando, sin tener en cuenta ningún tipo de vínculo común a todos los miembros de la muestra. Los multidimensionales 0 relacionales, permiten tener en cuenta de forma simultánea distintas variables obtenidas del estudio realizado (Sanz y Martín, 1998).

D ado que el objetivo de este trabajo es poner de manifiesto que la utilización de más de un método de recogida de datos permite validar y contrastar la información obtenida, vamos a ceñimos a aquellos indicadores que han podido ser extraídos por ambos métodos:

* Tipo de documento

* Títulos de revista

* Temática de las revistas

* Índice de aislamiento

* Capacidad idiomática de los investigadores

* Interdisciplinariedad de las Ciencias de la Vida 


\section{RESULTADOS Y DISCUSIÓN}

En los siguientes apartados vamos a centramos en los resultados obtenidos a partir de los indicadores señalados en el apartado de Metodología, mediante la utilización de ambos métodos, discutiendo dichos valores así como las discrepancias encontradas en algunos casos.

\section{Tipo de documento}

El valor del método indirecto (análisis de las demandas de documentos) estuvo dado por los valores porcentuales de los distintos tipos de documentos solicitados, mientras que en la encuesta se obtuvo del porcentaje de respuestas a la pregunta relativa a los tipos de documentos que más les interesaban para su trabajo.

Aunque solicitaban y consultaban distintos tipos de documentos, la mayor coincidencia porcentual se observó en el artículo de revista, con un 98.52\% para las demandas y un 100\% para la encuesta. Esta situación puede deberse a que, como ya se había observado desde los primeros estudios sobre necesidades de información de los científicos experimentales, se sigue prefiriendo a las revistas científicas especializadas como fuente de información formal, frente a cualquier otro tipo de documentos, como libros, actas de congresos, patentes, etc. Otros trabajos que han estudiado el tipo de documento utilizado por los investigadores en campos como Genética y Medicina (Martín y Sanz, 1996; Sanz, \&. al., 1998) han descrito resultados similares en cuanto a la preferencia por el artículo de revistas científicas. Sin embargo, para el resto de tipos documentales existió una gran discrepancia entre los valores aportados por cada método. Es probable que esto se deba a la propia naturaleza de las técnicas aplicadas, que en el fondo permiten determinar las posibles discrepancias entre lo que dicen consultar y lo que realmente revisan. En el caso de las peticiones de documentos, los investigadores solicitan aquellos que realmente necesitan para su investigación, mientras que en la encuesta, al tratarse de preguntas cerradas, podían marcar todos aquellos que pueden consultar en un momento dado, aunque luego no lo utilicen o no lo soliciten al centro de información.

\section{Títulos de revistas}

En el método indirecto, el estudio de esta variable se realizó sobre 42.825 peticiones de artículos de revista, integrados en 4.582 títulos diferentes. Pese a que el número medio de solicitudes fue de 9.35 artículos por revista, hubo seis títulos que fueron solicitados en más de 200 ocasiones. La tabla 1 recoge los títulos que recibieron más de 200 peticiones de artículos - que suponen en conjunto el 12.19\% de las solicitudes- y el porcentaje que éstas representan frente al total. 
Tabla 1

Títulos de las revistas que han recibido al menos 200 peticiones

\begin{tabular}{lcc}
\hline TÍTULO DE REVISTA & № DE PETICIONES & $\%$ \\
\hline BIOCHIM BIOPHYS ACTA & 597 & 1,39 \\
J BIOLOGICAL CHEMISTRY & 549 & 1,28 \\
J CHROMATOGRAPHY & 480 & 1,12 \\
ANAL BIOCHEM & 345 & 0,81 \\
NATL ACAD SCI USA PROC & 321 & 0,75 \\
COMP BIOCHEM PHYSIOL & 316 & 0,74 \\
BRAIN RESEARCH & 277 & 0,65 \\
NATURE & 272 & 0,64 \\
BIOCHEM J & 260 & 0,61 \\
BIOSCI BIOTECH BIOCHEM & 239 & 0,56 \\
BIOCHEM & 238 & 0,56 \\
BIOCHEM PHARMACOL & 238 & 0,56 \\
SCIENCE & 229 & 0,53 \\
ARCH BIOCHEM BIOPHYS & 224 & 0,52 \\
BIOCHEM BIOPHYS RES COMMUN & 216 & 0,50 \\
NEW YORK ACAD SCI ANNALS & 208 & 0,49 \\
CELL TISSUE RES & 206 & 0,48 \\
\hline
\end{tabular}

En este rubro, el método de la encuesta ofreció un resultado de 611 títulos distintos con muy diferentes frecuencias de uso, ya que mientras algunas revistas eran utilizadas por más de 70 usuarios, 346 títulos lo eran únicamente por uno. En la tabla 2 se indican los títulos consultados por más de 10 encuestados y el porcentaje de uso que supone frente al total. 
Tabla 2

Revistas más utilizadas y porcentaje de uso obtenido a partir de la encuesta

\begin{tabular}{lcc}
\hline TÍTULO DE REVISTA & № DE USUARIOS & $\%$ \\
\hline NATURE & 73 & 4,87 \\
SCIENCE & 43 & 2,87 \\
CELL & 31 & 2,07 \\
J BIOLOGICAL CHEMISTRY & 22 & 1,47 \\
PLANT PHYSIOLOGY & 22 & 1,47 \\
J BACTERIOLOGY & 20 & 1,34 \\
APPL AND ENVIRON MICROBIOLOGY & 17 & 1,13 \\
THEORETICAL APPLIED GENETIC & 17 & 1,13 \\
BRAIN RESEARCH & 14 & 0,93 \\
GENETICS & 13 & 0,87 \\
J IMMUNOLOGY & 13 & 0,87 \\
NATL ACAD SCI USA PROC & 13 & 0,87 \\
PLANTA & 12 & 0,80
\end{tabular}

Si comparamos los títulos que aparecen en ambas tablas, observamos que hay cinco coincidentes, y los porcentajes de artículos solicitados de tres de ellos son relativamente similares a los mencionados por los investigadores encuestados. La mayor diferencia se encuentra en el rango porcentual de las peticiones de documentos de estas revistas (tabla 1) que va del 1.39\% al 0.48\%, mientras que en la tabla 2 se observa que este rango es mucho más elevado, probablemente debido a la concentración que se produce al solicitarles en el cuestionario que indicaran únicamente los cuatro títulos más consultados.

La diferencia porcentual que se observa en la tabla 2 entre la utilización de las dos primeras revistas, Nature y Science, y el resto de los títulos, puede atribuirse a su carácter multidisciplinar que hace que sean consultadas por muchos investigadores; mientras que el resto de los títulos, al ser más especializados, son consultados únicamente por los científicos que trabajan en el área de investigación que les compete. La especialización de la ciencia implica la de sus medios de comunicación, situación que se manifiesta en los resultados, puesto que muestran que más de la mitad de los títulos son consultados únicamente por un investigador.

\section{Temática de las revistas}

La situación que acabamos de comentar se patentiza al realizar el estudio temático de las revistas que tiene como objetivo agruparlas por afinidades. La determinación temática de cada título se hizo mediante el repertorio ULRICH's (versión del verano de 1998 en CD-ROM). Para el análisis de peticiones de documentos se 
clasificaron temáticamente las 42.593 en las que se pudo localizar el título de la revista, y para la encuesta las 603 publicaciones localizadas.

Las demandas se encontraban distribuidas entre 4.415 revistas que a su vez se encuadraban en 79 temáticas diferentes, cada una con muy distinto número de documentos, pues mientras a Biología pertenecían casi 20.000 peticiones y más de 7.000 a Medicina, había 38 disciplinas con menos de diez documentos y 10 de las que se solicitó uno solo.

En la tabla 3 se muestra en orden descendente la frecuencia de las 10 temáticas que contenían al menos 75 títulos solicitados. La primera columna hace referencia a las temáticas, la segunda a la frecuencia de revistas solicitadas, y la tercera al porcentaje que supone el número de títulos de cada temática con relación al total.

Tabla 3

Temáticas que contienen al menos 75 títulos de revistas solicitadas

\begin{tabular}{lrr}
\hline TEMÁTICA & TOTAL DE REVISTAS & \multicolumn{1}{c}{$\%$} \\
\hline BIOLOGÍA & 1.315 & 29,78 \\
MEDICINA & 1.099 & 24,89 \\
AGRICULTURA & 289 & 6,55 \\
QUÍMICA & 225 & 5,10 \\
MULTIDISCIPLINAR & 191 & 4,33 \\
CIENCIAS GEOLÓGICAS & 169 & 3,83 \\
FARMACIA Y FARMACOLOGÍA & 150 & 3,40 \\
VETERINARIA & 120 & 2,72 \\
ESTUDIOS AMBIENTALES & 102 & 2,31 \\
ALIMENTOS E INDUSTRIA ALIMENTARIA 75 & 1,70 \\
\hline
\end{tabular}

Por otro lado, los 603 títulos de la encuesta se agrupaban en 25 temáticas, de las que la tabla 4 recoge las 10 que contenían al menos 9 títulos. La primera columna indica las temáticas, la segunda el número de revistas encuadradas en dichas temáticas, y la tercera el porcentaje que supone el número de revistas de cada temática con relación al total. 
Tabla 4

Clasificación temática de los títulos de revista más mencionados en la encuesta

\begin{tabular}{lcc}
\hline TEMÁTICA & TOTAL DE REVISTAS & $\%$ \\
\hline BIOLOGÍA & 300 & 49,75 \\
MEDICINA & 141 & 23,38 \\
AGRICULTURA & 37 & 6,14 \\
ESTUDIOS AMBIENTALES & 15 & 2,49 \\
PALEONTOLOGÍA & 15 & 2,49 \\
CIENCIAS GEOLÓGICAS & 14 & 2,32 \\
VETERINARIA & 11 & 1,82 \\
FARMACIA Y FARMACOLOGÍ & 10 & 1,66 \\
MULTIDISCIPLINAR & 9 & 1,49 \\
QUÍMICA & 9 & 1,49 \\
\hline
\end{tabular}

Pese al diferente número de temáticas encontrado por ambos métodos - debido fundamentalmente a la diferencia de títulos de revista con los que se trabajaba- existe una gran concordancia, puesto que en ambos casos más del 50\% de los títulos se agrupan sólo en dos temáticas, Biología y Medicina. La tercera temática es A gricultura, variando mínimamente los porcentajes encontrados por ambos métodos, al igual que ocurre con Medicina o Estudios Ambientales. Dado que 9 de las 10 primeras temáticas son coincidentes en las tablas correspondientes a los dos métodos, se puede concluir que son las de mayor interés para estos investigadores.

\section{Índice de aislamiento}

El interés de conocer este indicador radica en la posibilidad de estimar la cantidad de literatura nacional que consumen los usuarios, lo cual requiere conocer la nacionalidad de las revistas que solicitan y/ o utilizan.

En el rubro de peticiones de documentos se analizó la nacionalidad de 4.415 títulos diferentes, y de 603 en la encuesta, asignándose a cada revista la nacionalidad que el ULRICH's indicaba.

Las revistas de las que se solicitaban artículos mostraban 83 nacionalidades. En la tabla 5 recogemos la procedencia de aquellos títulos que recibieron un mínimo de 40 peticiones, y que en conjunto suponen más del 80\% de los mismos. 
Tabla 5

Nacionalidad de las revistas que publican los documentos solicitados en frecuencia absoluta y porcentaje

\begin{tabular}{llc}
\hline NACIONALIDAD & FREC ABS & $\%$ \\
\hline ESTADOS UNIDOS & 1347 & 30,51 \\
REINO UNIDO & 683 & 15,47 \\
ALEMANIA & 315 & 7,13 \\
HOLANDA & 265 & 6,00 \\
FRANCIA & 258 & 5,84 \\
ESPAÑA & 195 & 4,42 \\
JAPÓN & 149 & 3,37 \\
ITALIA & 148 & 3,35 \\
SUIZA & 102 & 2,31 \\
CANADÁ & 68 & 1,54 \\
IRLANDA & 42 & 0,95 \\
\hline
\end{tabular}

D e esta tabla se deduce directamente que el índice de aislamiento referido a las revistas que utilizan estos científicos es de $4.42 \%$, dado que es el porcentaje de fuentes españolas que solicitan.

En el caso de la encuesta, las 603 publicaciones proceden de 24 países distintos, pero en la tabla 6 sólo recogemos aquellos que publican más de 25 de estos títulos.

Tabla 6

Nacionalidad de las revistas que publican los documentos más mencionados en la encuesta en frecuencia absoluta y en porcentaje

\begin{tabular}{lcl}
\hline NACIONALIDAD & FREC ABS & $\%$ \\
\hline ESTADOS UNIDOS & 224 & 37,15 \\
REINO UNIDO & 143 & 23,71 \\
HOLANDA & 57 & 9,45 \\
ALEMANIA & 45 & 7,46 \\
ESPAÑA & 40 & 6,63 \\
FRANCIA & 25 & 4,15 \\
\hline
\end{tabular}

Como puede observarse, el valor del indicador obtenido por ambos métodos es muy similar, $4.42 \%$ y $6.63 \%$ respectivamente, y las diferencias pueden deberse al diferente número de títulos que se manejan en cada caso. Las 40 revistas españolas que consultan los investigadores españoles que trabajan en Ciencias de la Vida indican que el índice de aislamiento de es del 6.63\%, muy parecido al $6.3 \%$ obtenido 
por Vázquez y G onzalo (1997) - mediante un análisis de petición de documentos al CIND O C, para revistas españolas de Biología, Medicina y Agricultura- , valor que coincide casi exactamente con el nuestro.

\section{Capacidad idiomática}

El análisis indirecto de esta característica se ha realizado a partir del idioma en que se encontraban los documentos solicitados, y el directo a partir de las respuestas a la pregunta de la encuesta sobre los idiomas que utilizaban para obtener información.

En este caso, la mayor coincidencia se encuentra en la consulta de revistas en inglés, que es del 95.42\% y 99.75\% dependiendo del método aplicado. Los siguientes idiomas más utilizados según ambos métodos son, además del español que aparece en segundo lugar, el francés y el alemán. Si bien las frecuencias de documentos pedidos en estos idiomas no coinciden con las obtenidas a través de la encuesta, pues en ella además de tener preguntas cerradas en las que se indicaban los idiomas más frecuentemente usados, podían añadir otros distintos, lo que hace que aparezcan muchos más idiomas, y con distintas frecuencias, que los encontrados a través de las peticiones. En cualquier caso ambos resultados son coincidentes con los obtenidos en otros trabajos realizados en colectivos similares (Camí, đ. al., 1993).

\section{Interdisciplinariedad}

El análisis de la relación entre las distintas temáticas que participan en Ciencias de la Vida, así como de los centros de investigación españoles, informa sobre la interdisciplinariedad existente en esta área científica. Para llegar a ese punto se ha utilizado el análisis de correspondencias, cuya representación gráfica (mapa) constituye un indicador multidimensional o relacional. Con los datos obtenidos de las peticiones de documentos al CIND oc se obtuvo el mapa representado en la figura 1, y con los de la encuesta el de la figura 2.

Dada la gran cantidad de centros y temáticas diferentes para la realización del mapa de la figura 1 sólo se tuvieron en cuenta los centros que habían realizado más de 500 peticiones al CIND o c y las temáticas que éstos habían solicitado al menos en 300 ocasiones. Los nombres completos de las etiquetas correspondientes a los centros y temáticas que aparecen en esta figura se encuentran desarrollados en el apéndice 1. 
Figura 1

Asociación entre centros $(\boldsymbol{\Delta})$ y temáticas $(\bullet)$ obtenida

a partir de las revistas que han solicitado al CINDOC

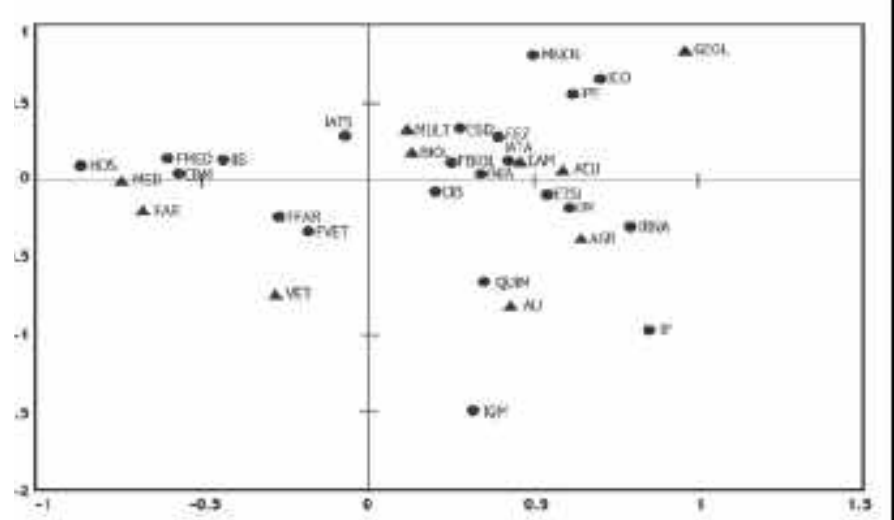

En la elaboración del mapa de la figura 2 se consideraron sólo aquellos centros de investigación en Ciencias de la Vida donde se encuestó a los científicos. D e esta aplicación se recibieron al menos 15 respuestas, más las temáticas mencionadas al menos por 5 de ellos. Los nombres completos de las etiquetas correspondientes a los centros y temáticas que aparecen en la figura se encuentran desarrolladas en el apéndice 2.

Figura 2

Asociación entre centros de investigación ( $\boldsymbol{\Delta}$ ) y temáticas a partir de datos obtenidos de la encuesta

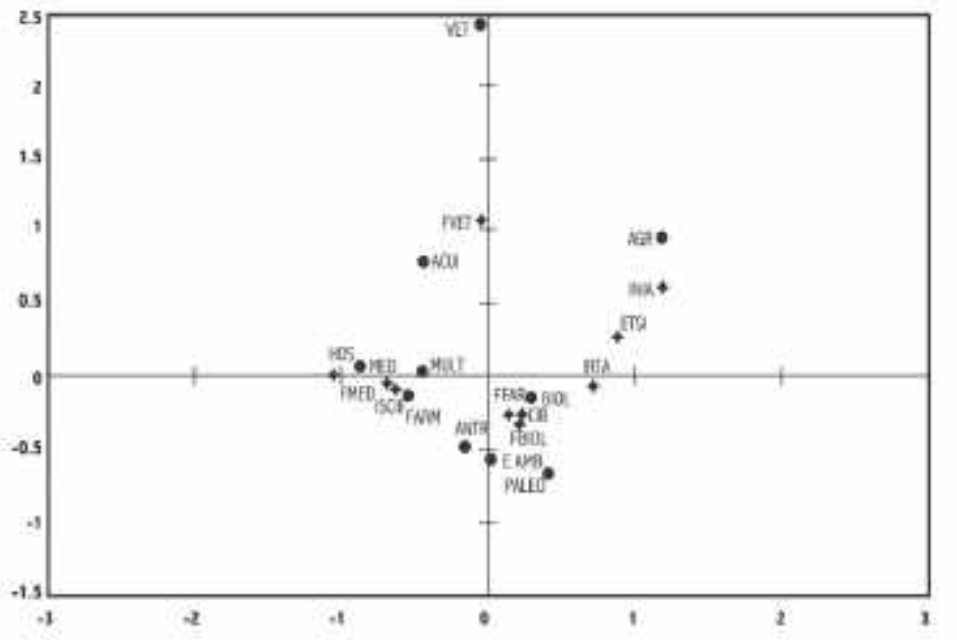


A pesar a las discrepancias causadas por el diferente número de datos sobre centros y temáticas con los que se ha trabajado en cada caso, en ambos mapas el primer eje, o eje de abscisas, quedó determinado por la contribución de la variable centros, y por tanto discrimina en función de sus diferencias o su especificidad. En los dos mapas se distinguen, por su especialidad, tres agrupaciones de centros de investigación: a la derecha los que trabajan en temas de agricultura y ganadería, a la izquierda los más directamente relacionados con la salud, y en el origen o sus cercanías, los menos especializados o más interdisciplinares.

En cuanto a la correspondencia que buscamos entre centros de investigación y temáticas, se observa que los centros del extremo derecho del eje forman un conjunto relativamente homogéneo, pues comparten la utilización de revistas sobre Agricultura (AGR). Los centros de investigación cercanos al origen emplean revistas de biología (BIOL), química (QUIM), estudios ambientales (EAMB), etc., y en ambos mapas, la temática Estudios Ambientales (EAMB) muestra una mayor asociación con las Facultades de Biología (FBIOL). Por otro lado, la proximidad al origen de dichos centros de investigación significa una relación equilibrada, con respecto a todas las temáticas, que refleja la interdisciplinariedad del trabajo que realizan, y, por tanto, la consulta de revistas de muy distintas temáticas. El razonamiento también es válido para las temáticas, es decir, aquellas situadas en torno al origen, fundamentalmente Biología (BIOL) y Multidisciplinar (MULT), presentan esa posición por el uso regular que hacen de ellas todos los centros de investigación estudiados. En el extremo izquierdo del eje, se observa una clara correspondencia entre los centros de investigación aquí situados y las revistas encuadradas en Medicina (MED) y Farmacia (FAR).

Probablemente la mayor diferencia entre ambos mapas está en la posición de las Facultades de Veterinaria (FVET), pues la que ocupa este centro en la figura 2 puede deberse a que en la encuesta, son los únicos centros de investigación que citan revistas especializadas en este tema (VET), a pesar de que utilizan publicaciones de muy distintas temáticas. Sería una situación similar a la del Instituto de Acuicultura Torre de la Sal (IATS) en la figura 1, cuyo comportamiento respecto al patrón general probablemente se deba a su especificidad, y aunque su colección sea adecuada en Acuicultura (ACUI), puede no serlo en otras temáticas, lo que obliga a sus investigadores a pedir por préstamo interbibliotecario documentos de temáticas distintas.

Podemos pues concluir que las principales diferencias entre ambos mapas se pueden deber al diferente número de centros de investigación que intervienen en ambos, pues el mayor rango de datos del método indirecto se traduce en un mayor número de centros y de temáticas.

Estas diferencias se minimizan si hacemos un nuevo análisis de correspondencias con los datos obtenidos en el análisis de peticiones de documentos, pero descartando algunos de ellos y considerando únicamente los centros de investigación que también aparecen en la encuesta. El mapa que se obtiene en este caso está 
representado en la figura 3, en la que puede observarse que la única diferencia existente entre las figuras 2 y 3 es que en la 2 aparece el Instituto de Salud Carlos III (ISCIII), mientras que en la figura 3 lo hace su homólogo, el Instituto de Investigaciones Biomédicas (IIB). Los nombres completos de las etiquetas correspondientes a los centros de investigación y temáticas que aparecen en esta figura se desarrollan en el apéndice 3.

Figura 3

Asociación entre centros de investigación coincidentes en ambos métodos ( $\Delta)$ y temáticas de las revistas $(0)$

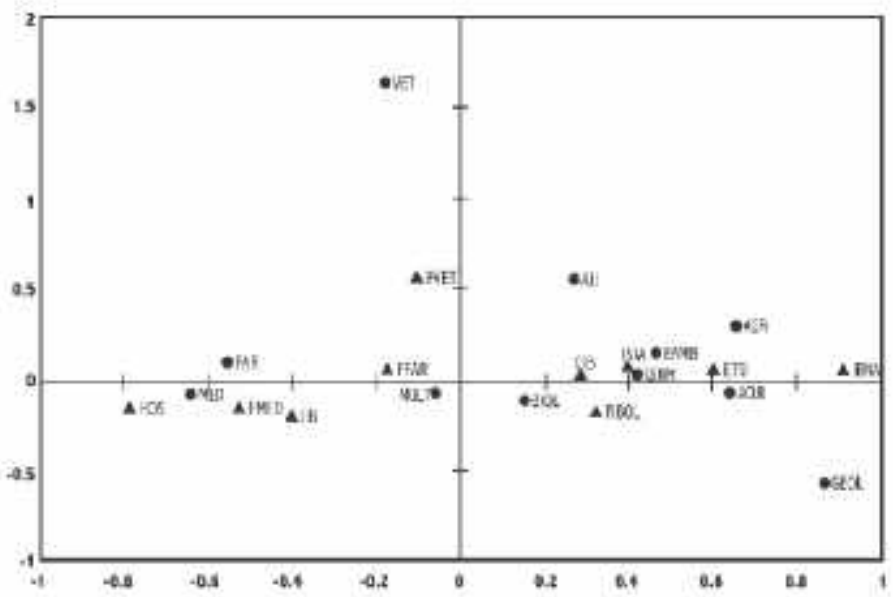

Pese a que la temática Acuicultura (ACUI) ocupa una posición completamente distinta, el hecho de disminuir el número de centros de investigación evita una gran cantidad de ruido. Las coincidencias observadas en los resultados de ambos métodos de recogida datos - mediante este indicador relacional- se ven muy claras, pues en los mapas de las figuras 2 y 3 se mantiene una disposición muy parecida de los centros, y de las temáticas que éstos solicitan y/ o mencionan en la encuesta.

La interdisciplinariedad de las líneas de investigación de algunos centros como las Facultades de Biología (FBIO L), les obliga a consultar literatura de muy diversas temáticas, por ello se sitúan en la proximidad al origen de coordenadas de los mapas realizados. Por otro lado, llama la atención que en todos los mapas las Facultades de Farmacia (FFARM) están relativamente alejadas de la temática que les es propia (FARM) y en cambio aparece una mayor relación con las Facultades de Medicina (FMED), el Instituto de Salud Carlos III (ISCIII), el Instituto de Investigaciones Biomédicas (IIB) y los Hospitales (HOS). Ello podría deberse a los estudios que realizan los investigadores, aunque también se explicaría a partir de los resultados obtenidos por Bordóns y Zulueta (1997) al estudiar la estructura y actividad 
científica de los grupos más productivos en Farmacia y Farmacología, pues observaron que el 70\% de los casos se encontraban trabajando en Facultades de Medicina (FMED).

\section{CONCLUSIONES}

La aplicación de diferentes técnicas en la recogida de datos ofrece un acercamiento más adecuado a la realidad que se quiere estudiar, además permite validar los resultados obtenidos por cada uno de los métodos utilizados, de ahí que hayan podido observarse grandes coincidencias en los valores de los indicadores.

Las dos técnicas confirmaron que las fuentes de información más utilizadas eran las revistas científicas y los libros. Tanto las revistas solicitadas al CIND 0 C, como las que dijeron consultar, pertenecían a muy diferentes temáticas, si bien en ambos casos el mayor porcentaje de los títulos se encuadraban en dos disciplinas: Biología y Medicina. El índice de aislamiento de estos investigadores es muy bajo, pues la literatura española que consultan presenta un promedio del 5\%.

No se ha observado barrera idiomática en estos científicos, pues aunque se observó que la información que solicitaban se encontraba fundamentalmente en inglés, también demandaron documentos en otros idiomas. A partir de la encuesta se comprobó que el 100\% de los investigadores utilizaba el inglés, y un gran porcentaje además manejaba información en otras lenguas.

Ambos métodos demuestran el carácter interdisciplinario de Ciencias de la Vida, puesto que los centros de investigación que realizan un estudio generalista mantienen mayor correspondencia con las temáticas multidisciplinares, aunque también con otras especializadas. Por el contrario, a pesar de la especificidad de los centros - en función de la temática de las revistas que más solicitaron y/ o mencionaron en la encuesta-, los científicos no dejaron de utilizar fuentes de información más generales.

Es importante señalar que las discrepancias encontradas en algunos de los indicadores seguramente se deben a los sesgos propios de cada uno de los métodos utilizados, hecho que ha sido señalado por varios autores. Por ello, creemos que el empleo conjunto de dos o más métodos evita dichas situaciones y mejora los resultados al hacerlos más válidos y fiables.

\section{BIBLIOGRAFÍA}

Allen, TH. J. "Information needs and uses". Anmual Reriewof Infomation ScieneandTedndogy, (1969), 4: 3-29.

BlanCHARD, L.; Giustini, D. "The 1997 Bibliotheca Medica Canadiana (BMC) survey: results, commentary and looking ahead". Biblidheea Medica Canadiana, (1997), 19 (1): 10-14. 
BORDÓNS, M.; Zulueta, M. A. "Comparison of research team activity in two biomedical fields". Säentomenics (1997), 40 (3): 423-36.

BRYMAN, A. Quantityandqualityinsodal rearch Londres: Unwin Hyman Ltd., (1988).

CAMí, J.; Fernández, M. T.; Gómez, I. “La producción científica española en biomedicina y salud. Un estudio a través del Science Citation Index (1986-1989)”. MedianaClínica(Barc.), (1993), 101 (19): 721-31.

CO OPER, M. "Perspectives on qualitative research with quantitative implications: Studies in information management". En:J. D. G LAZIER y R. R. POWELL (eds.) Qualitative reerch in information management. Englewood (Colorado): Libraries Unlimited, INC, (1992), p. 14-20.

CRAWFO RD, S. "Information needs and uses". Anmual Rexiewof Infomrtion SäenceandTedndog, (1978), 13: 61-81.

CRO NIN, B. CAB "Abstracts: A global view". A sibProceedings (1980), 32 (11/ 12): 425-37.

CHAU, M. Y. "Library services for international students: a study at Colorado State University". CdaradbLibraries (1994), 20 (3): 40-41.

DERVIN, B. "From de mind's eye of the user: The sense-making qualitative-quantitative methodology". En:J. D. G lazier y R. R. Powell (eds.) "Qualitative research in information management". Englewood (Colorado): LibraniesUnlimited INC, (1992), p. 61-84.

GLAZIER,J.D. "Qualitative and nonqualitative research methologies:Thesis, antithesis or synthesis?". En: J. D. G lazier y R. R. Powell (eds.) "Qualitative research in information management". Englewood (Colorado): LibrariesUnimited INC, (1992b), p. 201-14.

Herner, S.; Herner, M. "Information needs and uses in Science and Technology". Annual Reiewof InfomationSaieneandTehmolog, (1967), 2:1-34.

HERNON, P.; Schwartz, C. "Editorial: Need for more methodological and research design studies in library and information science". Libraryand Infomation SaieneReseren, (1994), 16 (1): 85-87.

JULIEN, H. "A content analysis of the recent information needs and users literature". LibraryandInfomationSaieneReserch (1996), 18 (1): 53-66.

MARTín Moreno, C. "Hábitosynecesidades deinformación delos científicos experimentales encuadrados en el área de las ciencias de la vida”. Tesis D octoral. Getafe, (Madrid): Universidad Carlos III, (1999), p. 375.

MARTín Moreno, C.; Sanz Casado, E. "Producción científica española en el área de genética” . ReuistaEspeñdadeDcumentacoónCientífica, (1996), 19 (4): 377-91. 
Menzel, H. "Information needs and uses in science and technology". Annal Reviewof Information Saienceand Techndogy, (1966), 1: 41-69.

PAISLEY, W.J. "Information needs and uses". Annual reviewof Information Saieneand Technogy, (1968), 3: 1-30.

SANZ Casado, E. Manual dectudiosdeusuarios Madrid: Fundación G ermán Sánchez Ruipérez, (1994).

SANZ Casado, E.; Martín Moreno, C. "Aplicación de técnicas bibliométricas a la gestión bibliotecaria”. InvesticadónBiblideedóǵca, (1998), 12 (24): 24-40.

SANZ Casado, E.; Suárez Balseiro, C.; G arcía Zorita, C. (1998). Estudio de la producción científica española en biomedicina durante el período 1991-1996. En Jornadas sobre documentación y ciencias médicas (Zaragoza, 23-25 O ctubre 1998). Zaragoza: Universidad deZaragoza, p. 97-121.

Siatri, R. "The evolution of user studies". Libni, (1999), 49 (3): 132-41.

SRID HAR, M. S. "Understanding the user - why, what and how?" . Library Saiene, (1995), 32 (4): 151-64.

Tillo tso N, J.; Cherry, J.; Clinton, M. "Internet use through the University of Toronto Library: D emographics, destinations, and users's reactions". Information TechndogyandLibraries (1995), 14 (3): 190-198.

VÁzQUEz Valero, M.; G onzalo Balmisa, Y. "Utilización de las revistas españolas a través de su demanda en un servicio de suministro de documentos". BdéíndelaANABAD, (1997), 47 (2): 193-203.

\section{APÉNDICES}

\section{Apéndice 1}

D escripción de las etiquetas de Centros y Temáticas que aparecen en la figura 1. Centros

CIB

$\mathrm{CBM}$

EBD

EEZ

ETSI

FBIOL

FFAR

FMED

FVET

HOS
Centro de Investigaciones Biológicas

Centro de Biología Molecular

Estación Biológica de D oñana

Estación Experimental del Zaidín

Escuelas Técnicas de Ingenieros Agrónomos y Agrícolas

Facultades de Biología y Ciencias

Facultades de Farmacia

Facultades de Medicina

Facultades de Veterinaria

Hospitales 


\begin{tabular}{ll}
\hline IATA & Instituto de Agroquímica y Tecnología de Alimentos \\
IATS & Instituto de Acuicultura Torre de la Sal \\
IF & Instituto del Frío \\
IIB & Instituto de Investigaciones Biomédicas \\
IIM & Instituto de Investigaciones Marinas \\
INIA & Instituto Nacional de Investigaciones Agrarias \\
IPE & Instituto Pirenaico de Ecología \\
IQM & Instituto de Química Médica \\
IRNA & Instituto de Recursos Naturales y Agrobiología \\
MNCN & Museo Nacional de Ciencias Naturales \\
Temáticas & \\
ACU & Acuicultura \\
AGR & Agricultura \\
ALI & Alimentos e Industria Alimenticia \\
BIOL & Biología \\
EAMB & Estudios Ambientales \\
FAR & Farmacia y Farmacología \\
GEOL & Geología \\
MED & Medicina \\
MULT & Multidisciplinar \\
QUIM & Química \\
VET & Veterinaria \\
\end{tabular}

\section{Apéndice 2}

D escripción de las etiquetas de Centros y Temáticas que aparecen en la figura 2. Centros

CIB Centro de Investigaciones Biológicas

ETSI Escuelas Técnicas de Ingenieros Agrónomos y Agrícolas

FBIOL Facultades de Biología y Ciencias

FFARM Facultades de Farmacia

FMED Facultades de Medicina

FVET Facultades de Veterinaria

HOS Hospitales

INIA Instituto Nacional de Investigaciones Agrarias

ISCIII Instituto de Salud Carlos III

Temáticas

IRTA Institut de Recerca y Tecnología de los Alimentos

ACUI Acuicultura

AGR Agricultura

ANTR Antropología

BIOL Biología

EAMB Estudios Ambientales

FARM Farmacia y Farmacología

MED Medicina

MULT Multidisciplinar

PALEO Paleontología

VET Veterinaria 


\section{Apéndice 3}

D escripción de las etiquetas de Centros y Temáticas que aparecen en la figura 3. Centros

CIB

ETSI

FBIOL

FFARM

FMED

FVET

HOS

INIA

IIB

IRNA

\section{Temáticas}

ACU

AGR

ALI

BIOL

EAMB

FAR

GEOL

MED

MULT

QUIM

VET
Centro de Investigaciones Biológicas

Escuelas Técnicas de Ingenieros Agrónomos y Agrícolas

Facultades de Biología y Ciencias

Facultades de Farmacia

Facultades de Medicina

Facultades de Veterinaria

Hospitales

Instituto Nacional de Investigaciones Agrarias

Instituto de Investigaciones Biomédicas

Instituto de Recursos Naturales y Agrobiología

Acuicultura

Agricultura

Alimentos e Industria Alimenticia

Biología

Estudios Ambientales

Farmacia y Farmacología

Geología

Medicina

Multidisciplinar

Q uímica

Veterinaria 